\title{
Antiphospholipid Syndrome Risk Evaluation
}

\author{
João Vilhena ${ }^{1}$, Henrique Vicente ${ }^{2}$, M. Rosário Martins ${ }^{3}$, José M. Grañeda ${ }^{4}$, \\ Filomena Caldeira ${ }^{4}$, Rodrigo Gusmão ${ }^{4}$, João Neves ${ }^{5}$ and José Neves ${ }^{6 *}$ \\ ${ }^{1}$ Departamento de Química, Escola de Ciências e Tecnologia, \\ Universidade de Évora, Évora, Portugal \\ jmvilhena@gmail.com \\ ${ }^{2}$ Departamento de Química, Centro de Química de Évora, Escola de Ciências e Tecnologia, \\ Universidade de Évora, Évora, Portugal \\ hvicente@uevora.pt \\ ${ }^{3}$ Departamento de Química, Instituto de Ciências Agrárias e Ambientais Mediterrânicas, \\ Escola de Ciências e Tecnologia, Universidade de Évora, Évora, Portugal \\ mrm@uevora.pt \\ ${ }^{4}$ Serviço de Patologia Clínica do Hospital do Espírito Santo de Évora EPE, Évora, Portugal \\ graneda1@sapo.pt, filomenacaldeiral@gmail.com, \\ dir.patcli@hevora.min-saude.pt \\ ${ }^{5}$ Drs. Nicolas \& Asp, Dubai, United Arab Emirates \\ joaocpneves@gmail.com \\ ${ }^{6}$ Centro Algorithmi, Universidade do Minho, Braga, Portugal \\ jneves@di.uminho.pt \\ * Corresponding author: phone: +351-934201337; fax: +351-253604471 \\ e-mail: jneves@di.uminho.pt
}

\begin{abstract}
The antiphospholipid syndrome is an acquired autoimmune disorder produced by high titers of antiphospholipid antibodies that cause both arterial and veins thrombosis as well as pregnancy-related complications and morbidity, as clinical manifestations. This autoimmune hypercoagulable state, often associated with coronary artery disease and recurrent Acute Myocardium Infraction, has severe consequences for the patients, being one of the main causes of thrombotic disorders and death. Therefore, it is extremely important to be preventive; being aware of how probable is to have that kind of syndrome. Despite the updated of the APS classification published as Sydney criteria, diagnosis of this syndrome remains challenging. Further research on clinically relevant antibodies and standardization of their quantification are required to improve clinical risk assessment in APS. This work will focus on the development of a diagnosis support system to antiphospholipid syndrome, built under a formal framework based on Logic Programming, in terms of its knowledge representation and reasoning procedures, complemented with an approach to computing grounded on Artificial Neural Networks.

The proposed model allowed to improve the diagnosis, classifying properly the patients that really presented this pathology (sensitivity about $92 \%$ ) as well as classifying the absence of APS (specificity ranging from $89 \%$ to $94 \%$ ).
\end{abstract}

Keywords: Antiphospholipid Syndrome · Logic Programming · Artificial Neural Networks · Knowledge Representation and Reasoning. 